\title{
Lasers de forte énergie et de forte puissance : vers les applications extrêmes
}

\author{
Christophe SIMON-BOISSON \\ Thales Optronique \\ christophe.simonboisson@fr.thalesgroup.com
}

Les lasers de très forte énergie et de très forte puissance moyenne sont par leurs caractéristiques mêmes des lasers extrêmes utilisés pour des applications extrêmes. À côté des énormes installations destinées à la production d'énergie, de nombreux lasers « à taille humaine » existent aujourd'hui dans les laboratoires, tirant parti de plusieurs découvertes fondamentales dans les technologies laser.

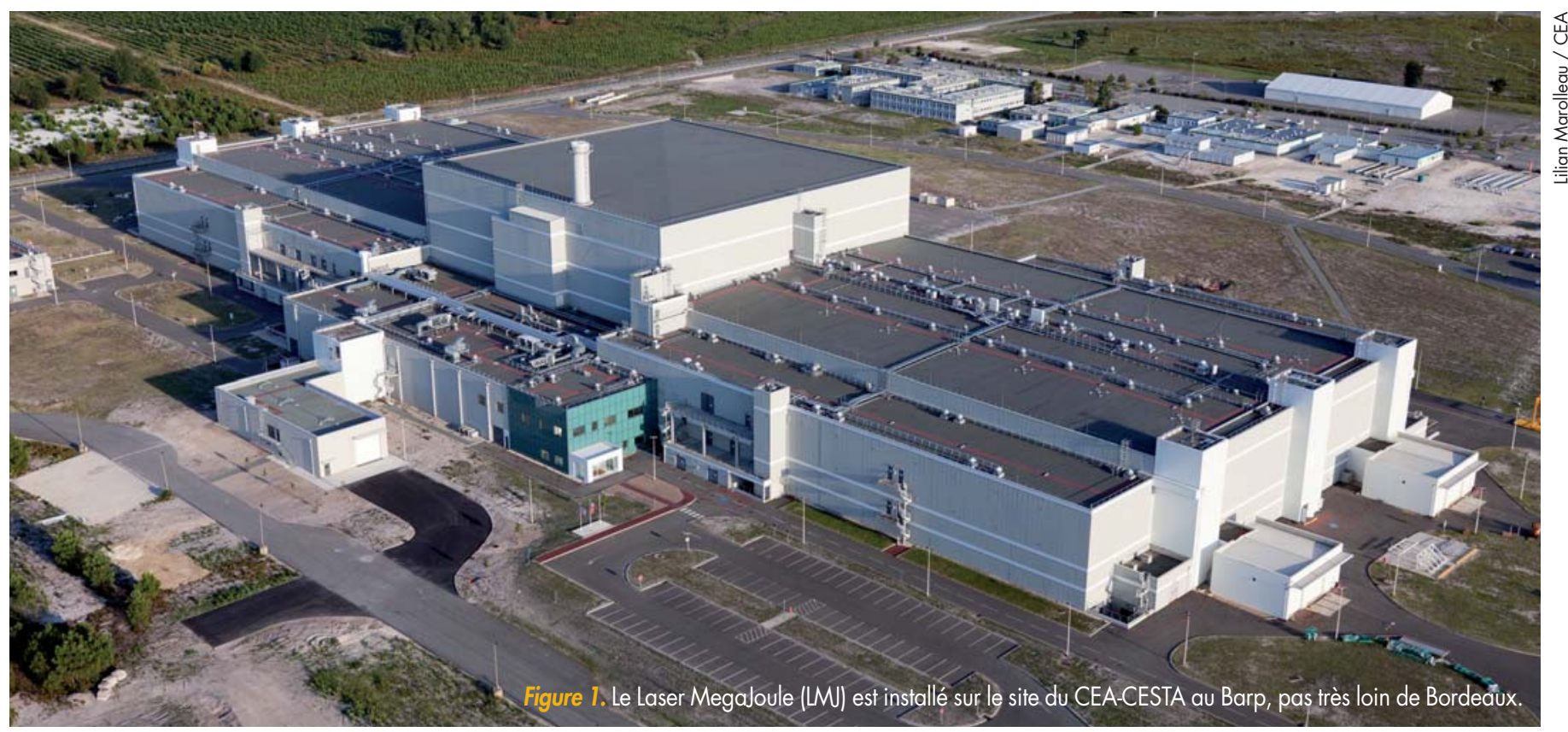

Les lasers extrêmes pour

la production d'énergie

\section{Des recherches qui datent du début du laser}

Très rapidement après la réalisation du premier laser en 1960 par Theodore Maiman, il a été envisagé d'utiliser le rayonnement laser pour réaliser la fusion nucléaire pour la production d'énergie dans le futur. La technologie actuelle de production d'énergie nucléaire civile utilise le mécanisme de fission de noyaux lourds. Cette technologie est aujourd'hui bien maîtrisée avec cependant des problématiques d'accès aux matières premières, de gestion des déchets radioactifs à durée de vie longue, et de sûreté. La production d'énergie par fusion nucléaire permettrait de résoudre ces problèmes si elle pouvait être mise en œuvre. La réaction de fusion nucléaire la plus favorable à la production d'énergie nucléaire est celle de la fusion entre les noyaux de deutérium et de tritium qui produisent des noyaux d'hélium et des neutrons. Il existe deux voies technologiques principales pour la fusion nucléaire pour la production d'énergie : la fusion magnétique qui est mise en œuvre dans le réacteur ITER en cours de construction et la fusion par confinement inertiel $(\mathrm{FCl})$ utilisant des lasers de très grande énergie. Le principe de la $\mathrm{FCl}$ consiste à comprimer en un temps très bref le mélange de deutérium-tritium contenu dans un microballon jusqu'à une densité de quelques centaines de grammes par centimètre cube, et le chauffer à une température de 10 millions de degrés. Des travaux sur la $\mathrm{FCl}$ ont démarré dès les années 60 principalement aux États-Unis, en Russie, en France et au Japon.

\section{Des installations pilotes aux systèmes opérationnels}

Les premières grandes installations ont été Nova à Livermore (USA) avec 10 faisceaux pour $60 \mathrm{~kJ}$ sur cible, Omega 
à Rochester (USA) avec 60 faisceaux pour 30 kJ, Phebus à Limeil (France) avec 2 faisceaux pour 6 kJ et Gekko à Osaka (Japon) avec 12 faisceaux pour $15 \mathrm{~kJ}$. Ces installations avaient pour vocation de faire progresser la compréhension des mécanismes physiques de la $\mathrm{FCl}$ et la technologie des installations mais n'étaient pas dimensionnées pour atteindre le break-even, c'est-à-dire produire plus d'électricité qu'elles n'en consommaient. C'est pourquoi il a été décidé, il y a une trentaine d'années aux USA et un peu plus tard en France, de construire des installations laser possédant les caractéristiques suffisantes pour démontrer en vraie grandeur la FCI. II s'agit de l'installation NIF (National Ignition Facility) basée à Livermore aux États-Unis et du Laser MegaJoule (LMJ) situé au centre CESTA du CEA au Barp à proximité de Bordeaux (figure 1). Dans les deux cas, le laser vient délivrer son énergie au microballon de deutérium-tritium par attaque indirecte (les faisceaux laser sont dirigés vers les parois internes du cylindre d'or contenant le microballon afin de produire un rayonnement $\mathrm{X}$ intense qui interagit avec le microballon) et la réaction de fusion est déclenchée par allumage par point chaud central.

\section{Des installations gigantesques en cours de mise en cuvre}

Ces grands lasers qui émettent dans I'ultra-violet (UV) à la longueur d'onde de $351 \mathrm{~nm}$ sont constitués d'un très grand nombre de faisceaux (192 pour le NIF, jusqu'à 240 pour le LMJ), qui sont tous focalisés en un même point de la cible de deutérium-tritium. Chaque faisceau, qui délivre une énergie de la classe $10 \mathrm{~kJ}$ dans I'UV est obtenu par conversions de fréquence successives (doublage et triplage de fréquence) dans des cristaux de KDP d'un faisceau initial infrarouge émettant à $1053 \mathrm{~nm}$. Ce faisceau est obtenu à partir d'un étage initial appelé pilote délivrant des impulsions d'énergie $1 \mathrm{~J}$ et dotées de propriétés particulières notamment sur le plan temporel et d'étages amplificateurs à disques de verre phosphate dopé au néodyme, pompés par des lampes flash et refroidis par air permettant de porter le niveau d'énergie à un niveau compris typiquement entre 15 et $20 \mathrm{~kJ}$. En raison des propriétés thermiques du verre phosphate et de la technologie de refroidissement, il n'est possible de réaliser que quelques tirs par jour, car il faut un temps important entre deux tirs laser pour que chaque disque de verre revienne à sa température d'équilibre.

Le NIF est opérationnel depuis 2009 et une première campagne d'essais pour l'obtention de la fusion (National Ignition Campaign) s'est déroulée de 2010 à 2012. Le break-even n'a pu être atteint mais des enseignements importants ont pu être tirés de cette campagne.

De son côté le LMJ est en cours de construction et la mise en service d'une première tranche de faisceaux est programmée pour fin 2014 permettant la réalisation de premières expériences en 2015.

Ces installations modernes se distinguent par leur gigantisme (quelques

\section{HORIBA \\ Scientific}

JOBIN YVON

Technology

\section{Réseaux de diffraction pour Laser ultra-intense}

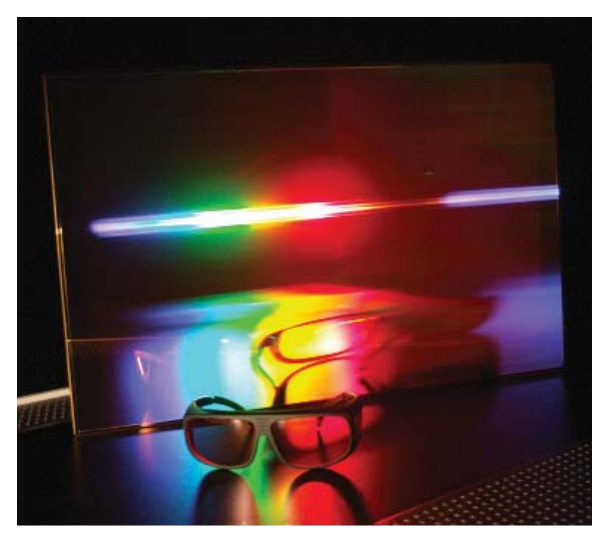

HORIBA Scientific, leader mondial des réseaux de diffraction pour laser femtoseconde, conçoit et fabrique une large gamme de réseaux pour laser depuis plus 30 ans :

$\checkmark$ Compression d'impulsions laser picoseconde et femtoseconde

$\checkmark$ Du proche Infra-Rouge à l'XUV

$\checkmark$ Du milli-Watt au multi-Petawatt

$\checkmark$ Holographie grand format : haute efficacité et homogénéité

$\checkmark$ Taille du réseau exceptionnelle jusqu'à 1 mètre

$\checkmark$ Haute tenue au flux laser

$\checkmark$ Conception sur mesure 
chiffres assez édifiants sur le NIF à titre d'exemple : le bâtiment occupe la superficie de trois stades de football américain ; le poids total de verre phosphate dans les lignes amplificatrices est de 129 tonnes, etc.) et leurs performances extrêmes (énergie record de 1,85 MJ d'UV atteinte en juillet 2012). Cela étant, la configuration de ces installations ne permet de disposer que d'un nombre limité de tirs laser à un coût très élevé par tir et le domaine couvert est essentiellement celui de la fusion nucléaire et plus généralement de la physique de la matière à haute densité d'énergie.

\section{Les lasers à haute puissance crête et haute intensité}

\section{Des découvertes fondamentales}

En parallèle de ces programmes de très grande envergure et des développements technologiques essentiels qu'ils ont suscité pour les lasers, d'autres découvertes se sont avérées capitales pour la technologie des lasers et le développement de leurs applications.

II s'agit de la mise en évidence du mode de fonctionnement des lasers en régime de cavité à modes verrouillés en phase permettant la génération d'impulsions laser ultra-brèves, de l'utilisation de cristaux de saphir dopé au titane (Peter Moulton en 1984) pour la génération et l'amplification des impulsions laser les plus brèves à l'heure actuelle, et enfin de la technique de l'amplification à dérive de fréquence (Gérard Mourou de Donna Strickland en 1985).

La combinaison de ces trois découvertes a concouru avec un succès sans cesse grandissant à la création d'un nouveau paradigme dans le domaine des installations laser à performances extrêmes, cette fois-ci dans le domaine des très hautes puissances crêtes et corollairement des très hautes intensités.

\section{Des performances en constante progression}

Les amplificateurs à dérive de fréquence (ou CPA pour chirped pulse amplification) au saphir titane voient leur performance en puissance crête multipliée par 10 tous les 5 à 6 ans. La puissance crête disponible a désormais dépassé le pétawatt, tant du point de vue des réalisations de la recherche académique (première publication d'un CPA saphir titane au pétawatt en 2010 par l'équipe Sud Coréenne de GISTAPRI) que de l'industrie (premier pétawatt commercial installé par Thales à Berkeley en juillet 2012).

Cet essor extraordinaire des systèmes haute puissance crête à base de saphir titane tient à plusieurs caractéristiques remarquables du saphir titane, au premier rang desquelles il faut relever la très grande largeur spectrale de la bande de gain et donc la capacité à produire des impulsions extrêmement courtes (aussi basses que 20 à 25 femtosecondes à comparer aux solutions à verre qui ne peuvent permettre de descendre qu'à 150-200 femtosecondes dans le cas le plus favorable du mélange de verres), la forte conductivité thermique de ce cristal qui permet le fonctionnement à des taux de répétition relativement «élevés » par rapport aux verres (jusqu'à $10 \mathrm{~Hz}$ pour des systèmes 200 térawatt et jusqu'à $1 \mathrm{~Hz}$ pour un système pétawatt) ce qui est extrêmement favorable aux expériences qui peuvent ainsi être reproduites beaucoup plus souvent. Le fait que le saphir titane puisse être pompé par des lasers visibles est également un facteur positif car les amplificateurs concernés ont un bon rendement (pouvant aller jusqu'à $45 \%$ ), et les lasers de pompage sont des produits commerciaux standard facilement disponibles sur le marché - et de mise en œuvre assez simple.

\section{Des installations accessibles à de nombreux laboratoires}

Ces progrès technologiques constants ainsi que le fait que ces installations restent à « dimension humaine » (il faut compter un laboratoire de quelques centaines de mètres carrés pour un système CPA saphir titane de classe pétawatt) et relativement simples à opérer (des équipes de quelques personnes sont suffisantes pour exploiter un système pétawatt saphir titane), font que de nombreux laboratoires se sont équipés de lasers de ce type, sont en train de le faire ou le projettent à court terme, soit en le réalisant par leurs propres moyens, soit dans la majorité des cas désormais, en en confiant la réalisation à un industriel du laser.

\section{La France aux premières loges}

Cette quête de la haute intensité a donc suscité la création de tout un écosystème dans lequel la France occupe une position d'excellence tant sur le plan industriel - ensembliers laser comme Thales et Amplitude ; fournisseurs de composants stratégiques (réseaux de compression de Horiba Jobin-Yvon, cristaux non-linéaires de Cristal Laser, miroirs déformables de ISP Systems et CILAS, composants optiques de grande taille de Thales SESO et Sagem Reosc) ; fournisseurs d'instrumentation (analyseurs de front d'onde et boucles d'optique adaptative de Phasics et Imagine Optic, mesureurs de durée et de phase spectrale de FastLite) - que sur le plan de la recherche académique:CEA à Saclay et au Barp ; laboratoires du plateau de Saclay comme le LOA, le LULI et I'IOGS; laboratoire CELIA de Bordeaux - qui a démarré très tôt sur le sujet et bénéficié de l'apport de chercheurs de classe mondiale. Mais c'est surtout la capacité de ces deux univers, académique et industriel, à interagir et à collaborer efficacement qui a été un levier décisif dans les succès passés et présents de notre communauté et qui devra perdurer et même se renforcer à l'avenir, dans un contexte de compétition internationale croissante, afin de remporter de futurs succès.

\section{Des applications en constante augmentation}

Il est en effet permis de penser que la dynamique des lasers de très haute intensité va se poursuivre et s'amplifier dans les prochaines années au regard des applications toujours plus nombreuses de ces lasers, potentiellement bien au-delà de la seule recherche scientifique.

\section{De l'accélération des particules aux applications médicales}

Si initialement, les premiers systèmes CPA de classe pétawatt ont été développés sur les très grandes installations 
de fusion comme Nova à Livermore (premier laser pétawatt réalisé, à base de verre phosphate, utilisant un bras de Nova modifié) dans la perspective de nouvelles techniques au service de la fusion nucléaire comme l'allumage rapide (c'est également l'objet du projet PETAL, qui est un laser en cours de construction au CEA en utilisant un bras amplificateur du LMJ pour une mise en service en 2015, et du grand projet Européen HiPER proposé initialement par le Royaume-Uni qui en est au stade des études préliminaires), les systèmes CPA à base de saphir titane ont permis des avancées extraordinaires dans le domaine de l'accélération de particules (électrons, protons, ions) avec la mise en évidence de nouveaux mécanismes d'accélération, comme l'accélération de sillage (Laser Wakefield Acceleration) dont le principe a été découvert dès 1979 par Tajima et Dawson. Cette nouvelle génération d'accélérateurs laser-plasma permet d'accélérer sur des distances relativement courtes (quelques dizaines de centimètres voire quelques mètres) des particules aux mêmes énergies que celles atteintes dans des accélérateurs linéaires de plusieurs kilomètres. Il est ainsi envisagé, pour les installations de protonthérapie permettant des traitements de certains cancers moins invasifs pour les patients, de remplacer les synchrotrons des installations actuelles (très coûteux et très encombrants ce qui limite le nombre des installations dans le monde à une trentaine, soit un niveau très insuffisant pour satisfaire les demandes) par des lasers pétawatt.

\section{Utiliser les rayonnements secondaires}

Avec ces particules accélérées par laser, outre les applications directes, il est également possible de générer de nouveaux rayonnements appelés alors rayonnements secondaires, en particulier des rayonnements $X$ et Gamma. II est par exemple envisageable d'utiliser ce type de sources qui par leur principe de fonctionnement présentent des résolutions spatiales très supérieures aux sources conventionnelles pour réaliser de la radiographie haute résolution dans le domaine du contrôle non destructif de pièces critiques, en particulier dans le domaine nucléaire et le domaine aéronautique.
D'autres projets sont déià lancés

\section{Le projet ELI}

Le domaine des lasers de haute intensité continue néanmoins d'évoluer avec la même vigueur et devrait continuer à le faire dans les prochaines années.

Le professeur Gérard Mourou, à son retour des États-Unis en 2005 pour prendre la direction du Laboratoire d'optique appliquée (LOA) a lancé l'initiative ELI (Extreme Light Infrastructure) dont l'objet initial était de réaliser une très grande infrastructure basée sur un laser exawatt, plus précisément 200 pétawatt. Le projet initial a finalement été séparé en quatre piliers dont trois sont déjà lancés et financés principalement au travers des fonds structuraux de l'Union Européenne :

- ELI Beamlines en République Tchèque, qui a démarré en 2011,

- ELI Nuclear Physics en Roumanie qui a démarré en 2012,

ELI Attoseconde en Hongrie qui a démarré en 2013.

Le quatrième pilier reste pour l'instant mis en attente jusqu'en 2015 et mettra en œuvre un très grand laser de classe 100 pétawatt dont la technologie dépendra des résultats observés sur les trois premiers piliers.

Le projet ELI République Tchèque a choisi de développer quatre lignes indépendantes, chacune équipée d'un laser différent. La quatrième et dernière ligne dont la construction n'est pas encore engagée sera constituée à partir d'un laser de 10 pétawatt très énergétique c'est-àdire délivrant au moins $1,5 \mathrm{~kJ}$, et va donc privilégier la technologie verre.

À l'inverse, le projet ELI Roumanie s'appuie sur la construction d'un laser unique délivrant deux faisceaux de 10 pétawatt. La réalisation de ce laser par Thales a démarré à l'été 2013 pour une fourniture en 2017. Il sera basé à nouveau sur la technologie du saphir titane et les impulsions seront délivrées à la cadence de 1 tir par minute. L'existence de sorties intermédiaires de plus faible niveau (100 TW et $1 \mathrm{PW}$ ) sur les bras $10 \mathrm{PW}$ permettront d'alimenter un grand nombre d'expériences.

Le projet ELI Hongrie, enfin, comportera plusieurs sources laser, sans atteindre toutefois la puissance crête de 10 PW.
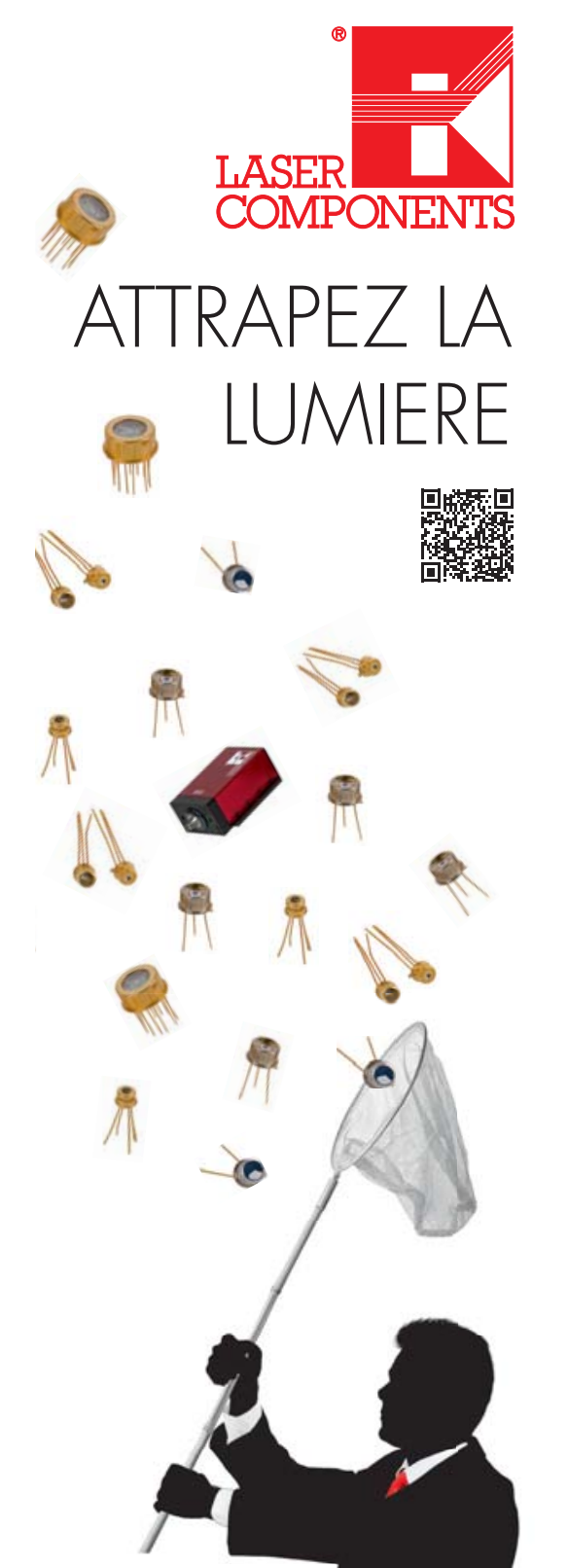

Photodiodes

Avalanche

Si-APDs
- $\lambda=260-1100 \mathrm{~nm}$

$\lambda=260-1100 \mathrm{~nm}$
$\varnothing=230 \mu \mathrm{m}-3 \mathrm{~mm}$

InGaAs APDs

- $\lambda=1000-1650 \mathrm{~nm}$

- $\varnothing=80 \mu \mathrm{m}, 200 \mu \mathrm{m}$ $350 \mu \mathrm{m}$

Récepteurs APD

- Versions Si et InGaAs

- DC- $25 \mathrm{MHz}$

- $\mathrm{AC}-700 \mathrm{MHz}$

APD Spécifiques

- APD pour comptage

de photon

- Versions refroidies

Versions avec

filtres intégrés

Versions sur-mesure

- APDs couplés sur fibre 


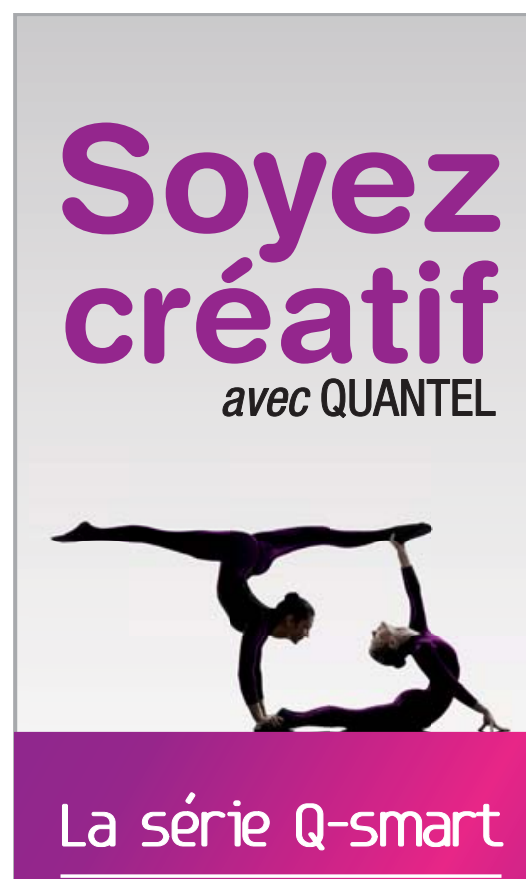

Les nouveaux lasers Nd:YAG pulsés par Quantel

- Ergonomie

- Optimisation automatique de l'énergie

- Mise en route rapide

\section{Q Quantel}

quantel@quantel-laser.com www.quantel-laser.com

\section{Le projet APOLLON}

II faut souligner que dans la mouvance de ces projets ELI, un projet précurseur a été lancé en France dès 2007. Il s'agit du projet APOLLON qui mettra en œuvre un laser de 10 pétawatt principalement basé sur un CPA à saphir titane, délivrant à la cadence de 1 tir par minute des impulsions dont l'énergie sera comprise entre 150 et 200 joules, et la durée entre 15 et 20 femtosecondes.

Cette installation sera accueillie en 2014 dans un bâtiment du CEA en cours d'aménagement sur le site de l'Orme des Merisiers et devrait entrer en service courant 2015.

\section{Le projet XCELS}

En parallèle des projets ELI s'est engagé en Russie un très gros projet nommé XCELS lancé dans le cadre de l'opération « Mega Science ». Ce projet consistera à construire un très grand laser de 180 pétawatt, donc pouvant être considéré comme de classe exawatt, constitué de 12 faisceaux délivrant chacun 15 PW. La technologie privilégiée dans les premières études est celle de I'OPCPA (optical parametric chirped pulse amplifier) basée sur l'amplification paramétrique dans des cristaux non linéaires pompés par des lasers de grande énergie.

Ce programme devrait démarrer à I'horizon 2015-2016 pour une réalisation du laser achevée autour de 2020.

\section{Déjà de nouvelles initiatives}

\section{L'initiative IZEST}

Ces différents projets sont d'ores et déjà engagés (les trois premiers piliers ELI) ou devraient l'être d'ici 1 à 2 ans (XCELS, quatrième pilier ELI). Au-delà, des initiatives permettant de préparer la génération suivante de sources laser et d'expériences de physique ont d'ores et déjà pris corps sous l'impulsion du professeur Mourou. L'initiative IZEST (International Zetawatt Exawatt Science \& Technology) vise d'une part à reculer sans cesse les frontières de la physique extrême, et d'autre part à développer des sources laser encore plus intenses en exploitant par exemple de nouveaux concepts d'amplification dans des plasmas.

\section{Explorer de nouvelles voies technologiques}

Une autre initiative consiste à explorer une voie technologique radicalement différente pour la technologie des sources laser. Nous avons vu que les premiers grands lasers avaient un taux de répétition extrêmement faible. Le choix du saphir titane comme matériau amplificateur laser a certes permis de lever en partie cet inconvénient mais pas complètement et le fait de pomper ces cristaux par des lasers pompés par flash conduit à des rendements énergétiques globaux qui restent très faibles, en deçà du pourcent. Les besoins grandissants en puissance crête tout comme en taux de répétition ne pourront clairement plus être satisfaits en conservant cette technologie car elle conduirait à des niveaux de consommation électrique et de dissipation thermique insoutenables avant de se heurter purement et simplement à un problème de faisabilité.

L'alternative crédible est la technologie des lasers et amplificateurs à fibre optique qui ont un rendement énergétique élevé et permettent une bonne gestion des effets thermiques. II y a cependant deux inconvénients, I'un relatif aux durées d'impulsion minimales accessibles qui se limite à quelques centaines de femtosecondes, ce qui permet néanmoins de répondre à de nombreux besoins, l'autre causé par l'apparition précoce d'effets non linéaires liés au confinement important du rayonnement dans le cœur de la fibre optique, effets qui perturbent le fonctionnement du laser voire endommagent la fibre de manière irréversible. Ce second inconvénient peut être évité en réalisant la combinaison cohérente d'un très grand nombre d'amplificateurs à fibre. Des premières analyses sur le sujet ont été menées dans le cadre du réseau thématique ICAN (International Coherent Amplification Network) financé par l'Union Européenne et dont les activités se sont déroulées de février 2012 à juillet 2013. Des suites à cette initiative extrêmement prometteuse sont d'ores et déjà à l'étude : gageons que cette nouvelle technologie de sources laser étendra encore davantage le champ des applications possibles. 